\title{
INFLUENCE OF ION BOMBARDMENT ON MICROCRYSTALLINE SILICON MATERIAL QUALITY AND SOLAR CELL PERFORMANCES
}

\author{
G. Bugnon, A. Feltrin, F. Sculati-Meillaud, J.Bailat, Ch. Ballif \\ Institute of Microtechnology (IMT) - University of Neuchâtel \\ Rue A.-L.Breguet 2, CH-2000 Neuchâtel, Switzerland / e-mail: gregory.bugnon@unine.ch
}

\begin{abstract}
Microcrystalline hydrogenated silicon growth with VHF-PECVD was examined in an industrial type parallel plate $\mathrm{KAI}^{\mathrm{TM}}$ reactor. The influence of pressure on material quality was studied in single junction solar cells. Solar cells with their intrinsic layer prepared at higher pressures exhibit remarkable improvements, reaching $8.2 \%$ efficiency at 3.5 mbar. Further analyzes showed that $\mu \mathrm{c}$ Si:H intrinsic layers grown at higher pressures have a significantly lower defect density. These results are attributed to lower ion bombardment energies due to higher working pressures, which improve the microcrystalline material quality. Layer amorphization with increasing power density is observed at low pressure. Calculations show that the average ion energy drops from roughly $20 \mathrm{eV}$ to a few $\mathrm{eV}$ in the pressure range studied.
\end{abstract}

\section{INTRODUCTION}

Thin-film deposition of microcrystalline silicon ( $\mu \mathrm{c}$ $\mathrm{Si}: \mathrm{H})$ has attracted a lot of attention during those last years. It is of particular interest in the case of photovoltaic applications compared to amorphous silicon (a-Si:H) because of its higher robustness to light-induced degradation [1] while providing an enhanced absorption in the near-infrared part of the spectrum. For those reasons, $\mu \mathrm{c}-\mathrm{Si}: \mathrm{H}$ has become a material of choice in the development of highly efficient tandem solar cells when used with an a-Si:H top-cell.

However, many efforts are still to be made in order to improve overall understanding of the $\mu \mathrm{c}-\mathrm{Si}: \mathrm{H}$ growth by plasma-enhanced chemical vapor deposition (PECVD). For instance, because of its thick active layer requirement, $\mu \mathrm{c}-\mathrm{Si}: \mathrm{H}$ material has to be grown at high deposition rates while retaining high material quality (near the transition region from amorphous to microcrystalline silicon [2,3]). As of today, this seems to be the main concern for lowering industrial manufacturing costs.

A wide range of parameters have to be taken into account for this problem, such as hardware parameters: electrode geometry or shape, interelectrode distance, operating frequency, etc. and process parameters, easily adjustable for a given reactor, such as the power density, the pressure, the temperature and the gas flows or the actual gas composition in the process.
In this study, very high frequency PECVD (VHF$P E C V D)$ was used in a medium area $\mathrm{KAI}^{\mathrm{TM}}-\mathrm{S}$ reactor. Different process parameters and regimes for the growth of $\mu \mathrm{c}-\mathrm{Si}: \mathrm{H}$ thin-films were investigated. Structural material characterization of deposited $\mu \mathrm{c}-\mathrm{Si} \mathrm{H}$ layers is presented, and solar cells were prepared to emphasize the impact of the active layer on the solar cell electrical performances. Finally, Fourier Transform Photocurrent Spectroscopy (FTPS) was used to evaluate the defect density and the Urbach tail of the $\mu \mathrm{c}-\mathrm{Si}: \mathrm{H}$ intrinsic layer. A significant correlation of the intrinsic material quality with the solar cell efficiency is observed, and an interpretation in terms of ion bombardment energy is proposed.

\section{EXPERIMENTAL DETAILS}

\section{PECVD System}

The $\mathrm{KAI}^{\mathrm{TM}}$-S deposition system used for this study was developed by Oerlikon (formerly Unaxis). It consists of a closed plasma reactor (Plasmabox ${ }^{\circledR}$ ) contained within a main vacuum chamber. This configuration allows for a directional pumping from one of the plasma reactor's wall, which allows rapid plasma equilibration time, below the second [4]. Also the differential pumping between the two chambers limits possible cross contaminations. The deposition chamber is $47 \times 57 \mathrm{~cm}^{2}$ allowing substrates sizes up to $35 \times 45 \mathrm{~cm}^{2}$. A showerhead electrode is suspended at $2.5 \mathrm{~cm}$ from the grounded electrode. VHF power is supplied via a $40.68 \mathrm{MHz}$ generator (Advanced Energy CESAR ${ }^{\circledR} 4020400 \mathrm{~V}$ ), feeding the power through an automatic matching-box directly connected at the center of the electrode. Isothermal heating is obtained in the Plasmabox and deposition temperature was fixed at $180^{\circ} \mathrm{C}$ for all the depositions in this study.

\section{Raman crystallinity measurements}

Micro-Raman spectroscopy (Renishaw system 2000) was used to measure the crystallinity of the $\mu \mathrm{c}-\mathrm{Si}$ :H layers. The excitation wavelength was the emission line of an $\mathrm{Ar}^{+}$ laser at $514 \mathrm{~nm}$. The crystalline fraction $R_{c}$ was evaluated through the formula:

$$
R_{c}=\frac{I_{510}+I_{520}}{I_{480}+I_{510}+I_{520}},
$$

where $I_{x}$ is the integrated intensity under the Gaussian centered at $x \mathrm{~cm}^{-1}$. All measurements were carried out in 
air at room temperature. Thin-films were deposited on Schott AF 45 glass substrates, with a minimum $\mu \mathrm{c}-\mathrm{Si}: \mathrm{H}$ layer thickness of $150 \mathrm{~nm}$.

\section{Solar cells}

Standard $p-i-n$ solar cell were prepared to evaluate the different $\mu \mathrm{c}-\mathrm{Si}: \mathrm{H}$ layers. The front-contact was a textured zinc oxide $(\mathrm{ZnO})$ thin-film, grown on Schott AF 45 glass substrates, obtained through a modified lowpressure chemical vapor deposition (LPCVD) process developed at the IMT. The solar cells were then deposited using the $\mathrm{KAI}^{\mathrm{TM}}-\mathrm{S}$ reactor without breaking the pressure, with an intrinsic layer of approximately $1.2 \mu \mathrm{m}$ in thickness. These $i$-layers are grown on top of a thin $\mu \mathrm{c}-\mathrm{Si}: \mathrm{H}$ buffer layer above the $p$-layer. The patterned cells were $0.25 \mathrm{~cm}^{2}$ each, and the back contact was a $\mathrm{ZnO}$ thin-film grown using LPCVD as well, covered by a dielectric reflector. Current-voltage (I-V) curves were obtained using a WACOM AM1.5 solar simulator.

\section{Fourier-Transform Photocurrent Spectroscopy}

The material quality of the absorbing $\mu \mathrm{c}-\mathrm{Si}: \mathrm{H}$ layer incorporated in each cell was evaluated through FTPS measurements [5]. The absorption coefficient of lowenergy (sub-gap) photons gives important information on the electronic quality of the material: concentration of dangling bonds, i.e. defect density or recombination centers have a significant impact on the Urbach tail, and the signal at $0.8 \mathrm{eV}$ gives an estimation of the midgap defect density. The measurement being very sensitive to the type of $\mathrm{ZnO}$ used (variations of light diffusion and level of free carrier absorption induced by doping) a careful attention was given to make sure every cell was prepared using the same type of back- and front-contacts. Calibration of the absorption curves was performed at 1.35 $\mathrm{eV}$ at the corresponding absorption coefficient of crystalline silicon.

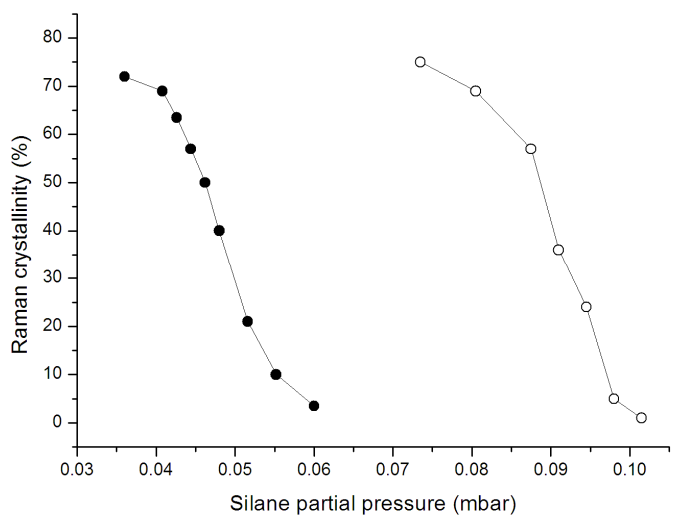

Fig. 1 - Transition from a-Si:H to $\mu \mathrm{c}-\mathrm{Si}: \mathrm{H}$ material as a function of silane partial pressure for two different regimes at $1.2 \mathrm{mbar}$ (filled circles) and 3.5 mbar (empty circles).

\section{RESULTS AND DISCUSSION}

\section{Amorphous to microcrystalline transition study}

Series of $\mu \mathrm{c}-\mathrm{Si}: \mathrm{H}$ layers were prepared at pressures $p$ of 1.2 and 3.5 mbar with varying silane concentration SC while keeping the total flow rate constant. SC is defined here in terms of the input flow rates as $\Phi_{\mathrm{SiH} 4} /\left(\Phi_{\mathrm{SiH} 4}+\right.$ $\left.\Phi_{\mathrm{H} 2}\right)$, where $\Phi_{x}$ is the input flow rate of $x$ in sccm. Fig. 1 shows the Raman cristallinity evolution of both regimes as a function of partial pressure of silane, defined as $p_{\mathrm{SiH} 4}=$ $p \times \mathrm{SC}$.

The transition zone from amorphous to microcrystalline silicon is observed. As expected, the increase of silane concentration in the discharge results in the growth of a predominantly amorphous phase of the material. Although the SC interval range is smaller at 3.5 mbar, once expressed in terms of partial pressure of silane, one can observe that the width of the crystallinity drop is nearly the same for both series, roughly $1.6 \times 10^{-2}$ mbar. This is an indication that silane depletion, which is low, is roughly the same in the two regimes [6].

\section{Solar cells performances}

Solar cells were deposited with their intrinsic layer prepared in those two regimes, near the transition region at roughly $50 \%$ crystallinity. The I-V curves from the best cells obtained in those two regimes are shown in Fig 2.

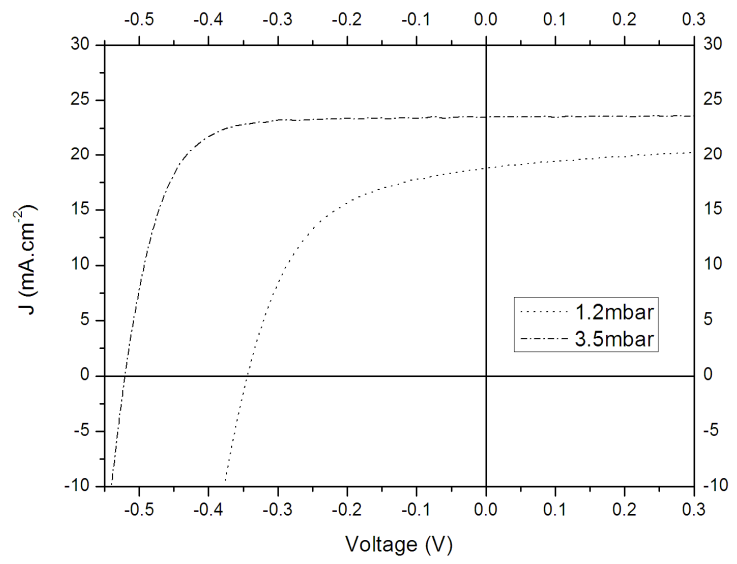

Fig. 2 - Current-voltage curves from microcrystalline $p-i-n$ cells prepared at 1.2 (dotted line) and 3.5 mbar (dashdotted line).

Remarkable improvements are observed for the cells prepared at higher pressure. Both the open-circuit voltage $\mathrm{V}_{\mathrm{oc}}$ and fill-factor $\mathrm{FF}$ are increased significantly, going from $0.34 \mathrm{~V}$ to $0.52 \mathrm{~V}$ and $52 \%$ to $72 \%$ respectively. The shortcircuit current density $\mathrm{J}_{\mathrm{sc}}$ increases as well from 18.8 to $22.2 \mathrm{~mA} . \mathrm{cm}^{-2}$. 


\section{Defect density of the intrinsic layer}

To further investigate this improvement, tests were carried out to analyze the material defect density. This was done through FTPS measurements, and the results can be seen in Fig. 3.

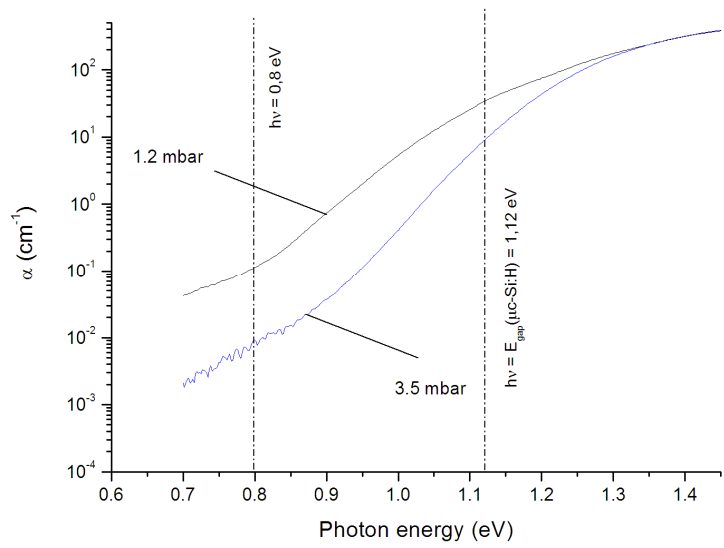

Fig. 3 - Sub band gap absorption coefficient obtained by FTPS measurements on $p-i-n \mu c-S i: H$ solar cells prepared at 1.2 and 3.5 mbar with the same $\mathrm{ZnO}$ as back and front contacts developed at the IMT.

The solar cell with its intrinsic layer prepared at 3.5 mbar shows a sub-gap absorption coefficient way below the $1.2 \mathrm{mbar}$ one. At $0.8 \mathrm{eV}$ more than one order of magnitude is observed, indicating that the defect density of the material is indeed significantly lowered when deposited at higher pressure. The correlation with the overall electrical cell performances is clear, leading to higher FF and $\mathrm{V}_{\mathrm{oc}}$ for low mid-gap defect density material.

Solar cells were deposited on other textured $\mathrm{ZnO}$ as well, and the same trend was observed, ensuring that this result is independent of the substrate used.

We interpret the difference of material quality observed with a varying pressure as an indication that ion bombardment energy plays an important role in the deposition process. As the deposition pressure is lowered, the capacitive sheaths become less collisional (i.e. ions mean free path increased), allowing more ions to impinge on the substrate's surface with higher energy, which in return is detrimental to the material quality.

\section{Raman cristallinity as a function of input power density}

In order to validate our interpretation the influence of power was also investigated in those two regimes. Starting with a SC giving a Raman crystallinity in the "transition zone", the power was then varied. The results are given in Fig. 4.
As the power density is increased, the two regimes exhibit quite different behaviors. At 3.5 mbar the increase in power density of the discharge results in a monotonous increase of the crystallinity, going from a complete amorphous phase at a low power of $350 \mathrm{~W}$, to a highly crystalline material at high power i.e. $79 \%$ at $650 \mathrm{~W}$. At the opposite, in the low-pressure regime the cristallinity first increases between 250 and $350 \mathrm{~W}$, going from $29 \%$ to $41 \%$, and then steadily decreases to a value of $27 \%$ at $550 \mathrm{~W}$.

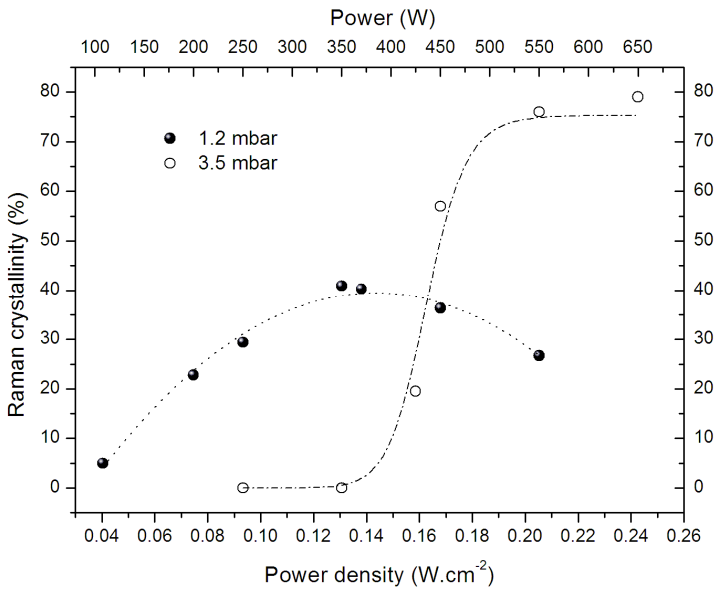

Fig. 4 - Raman crystallinity as a function of input power for both regimes, at 1.2 mbar (filled circles) and 3.5 mbar (empty circles). The dashed and dotted lines are a guide to the eye.

The significant differences observed here between those two regimes may be due to multiple factors, among which powder formation or ion bombardment energy seem to be the most reasonable in this context [7]. Still, the residence time being shorter in the 1.2 mbar regime (less than half) while having a lower silane partial pressure, the powder formation should be less promoted in this case. For this particular reason, it is suspected that the decrease in crystallinity observed at lower pressure is due to a stronger ion bombardment which can induce amorphization. At 3.5 mbar the capacitive sheaths being more collisional, it is expected that the ions impinging the substrate's surface tend to lose a larger portion of their kinetic energy.

\section{Systematic study of solar cell performance dependency with deposition pressure}

Multiple solar cell depositions were conducted in the regimes of $1.2,2.5$ and 3.5 mbar for their intrinsic layer, near the transition region.

The average efficiencies obtained in these regimes are given in Fig. 5. As can be seen, there is a clear trend indicating that deposition of $\mu \mathrm{c}-\mathrm{Si}: \mathrm{H}$ at higher pressure for the intrinsic layers of $p-i-n$ solar cells is beneficial for their 
overall performances. On the same figure the estimated average ion-bombarding energy $\varepsilon_{i}$ impinging the surface is indicated.

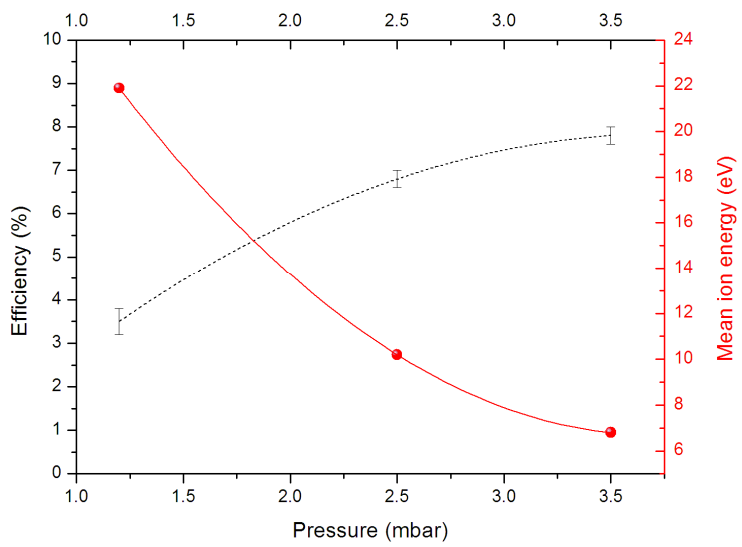

Fig. 5 - Average $\mu \mathrm{c}-\mathrm{Si}$ :H solar cell efficiency as a function of the deposition pressure for the intrinsic layer (error bars are standard error of the mean), the dotted line is a guide to the eye. The mean ion energy was calculated for each deposition regime (solid red line).

It is calculated in the case of collisional sheaths as

$$
\varepsilon_{i} \approx 0.62 \frac{\lambda_{i}}{s_{m}} \bar{V}
$$

where $\lambda_{i}$ is the mean free path, $s_{m}$ the sheath length and $V$ the time averaged voltage across the sheath [8].

The best cell was obtained at $3.5 \mathrm{mbar}$, with an efficiency of $8.2 \%$. It can be seen that the ion bombardment energy is scaling in the same range than the one already discussed by Kondo et al. [9], on which cristallinity is significantly affected, thus further supporting our interpretation.

\section{CONCLUSION}

Growth of $\mu \mathrm{c}-\mathrm{Si}: \mathrm{H}$ thin films was studied in large area $\mathrm{KAI}^{\mathrm{TM}}$-S VHF reactor to evaluate the importance of pressure on the material quality.

Single junction microcrystalline $p$-i-n cells were prepared with an intrinsic layer deposited at 1.2 and 3.5 mbar near the transition region, in low depletion regimes. For roughly the same cristallinity, it is observed that solar cells made at higher pressures exhibit significantly higher performances. FTPS measurements of both $\mu \mathrm{c}-\mathrm{Si}: \mathrm{H}$ intrinsic layers included in the solar cells concur with this observation: with increasing pressure the defect density is significantly lowered, which is an indication that the electronic material quality is getting better. $\mu \mathrm{c}-\mathrm{Si}: \mathrm{H}$ transitions measured as a function of RF power and calculations of the mean ion energy impinging on the substrate suggest that ion bombardment is likely to be responsible for these differences. A general trend was observed when comparing solar cells with their $i$-layer prepared at 1.2, 2.5 and $3.5 \mathrm{mbar}$, as the efficiencies were increasing along with the increase in pressure.

Further investigations will be pursued to evaluate more precisely the importance of ion bombardment energy in the $\mu \mathrm{c}-\mathrm{Si}: \mathrm{H}$ thin-film growth.

\section{ACKNOWLEDGMENTS}

The authors would like to thank B. Strahm, A. Howling and C. Hollenstein from the CRPP of Lausanne for useful discussions. Financial support by the Swiss Federal Office of Energy under contracts 101191, 153032 and by the EU-Project "ATHLET" under contract 019670 are acknowledged.

\section{REFERENCES}

[1] J. Meier et al., "Complete microcrystalline p-i-n solar cell - Crystalline or amorphous cell behavior?", Appl. Phys. Lett. 65, 1994, pp. 860.

[2] U. Kroll, et al., "From amorphous to microcrystalline silicon films prepared by hydrogen dilution using the vhf (70 mhz) gd technique", J. Non-Cryst. Solids 227-230, 1998, pp. 68-72.

[3] N. Wyrsch, et al., "Effect of the microstructure on the electronic transport in hydrogenated microcrystalline silicon", J. Non-Cryst. Solids 299-302, 2002, pp. 390-394.

[4] A. A. Howling, et al., "Fast equilibration of silane / hydrogen plasmas in large area RF capacitive reactors monitored by optical emission spectroscopy", Plasma Sources Sci. Technol. 16 (4), 2007, pp. 679-696.

[5] M. Vanecek and A. Poruba, "Fourier-transform photocurrent spectroscopy of microcrystalline silicon for solar cells", Appl. Phys. Lett. 80, 2002, pp. 719.

[6] B. Strahm, et al., "Plasma silane concentration as a determining factor for the transition from amorphous to microcrystalline silicon in $\mathrm{SiH} 4 / \mathrm{H} 2$ discharges", Plasma Sources Sci. Technol. 16 (1), 2007, pp. 80-89.

[7] B. Strahm, "Cost-Effective Production of Thin Film Microcrystalline Silicon for Solar Cells", 2007, EPFL PhD thesis.

[8] M. Liebermann, A. Lichtenberg, "Principles of Plasma Discharge and Material Processing", Second Edition, 2005, Wiley, pp. 411.

[9] M. Kondo, "Microcrystalline materials and cells deposited by RF glow discharge", Sol. Energy Mat. \& Sol. Cells 78,,2003, pp. 543-566. 